\title{
Effects of Curcumin on Cognitive Function-A Systematic Review of Randomized Controlled Trials
}

\author{
Nathan Seddon ${ }^{1, \#}$, Nathan M. D’Cunha',2,\#, Duane D. Mellor ${ }^{2}$, Andrew J. McKune ${ }^{1,2,3,4}$, \\ Ekavi N. Georgousopoulou $2,5,6,7$, Demosthenes B. Panagiotakos ${ }^{1,2,5,8}$, \\ Jane Kellett ${ }^{1,2}$ and Nenad Naumovski ${ }^{1,2^{*}}$
}

\begin{abstract}
${ }^{1}$ Faculty of Health, University of Canberra, Canberra, Australia; ${ }^{2}$ Collaborative Research in Bioactive and Biomarkers Groups (CRIBB), University of Canberra, Bruce, ACT, Australia; ${ }^{3}$ Discipline of Sport and Exercise Science, Research Institute for Sport and Exercise, Faculty of Health, University of Canberra, Canberra ACT, Australia; ${ }^{4}$ Discipline of Biokinetics, Exercise and Leisure Sciences, School of Health Sciences, University of KwaZulu-Natal, Durban, KwaZulu-Natal, South Africa; ${ }^{5}$ Department of Nutrition-Dietetics, School of Health Science and Education, Harokopio University, Athens, Greece; ${ }^{6}$ Medical School, Australian National University, Canberra, ACT, Australia; ${ }^{7}$ School of Medicine Sydney, The University of Notre Dame, Sydney, Australia; ${ }^{8}$ Department of Kinesiology and Health at The

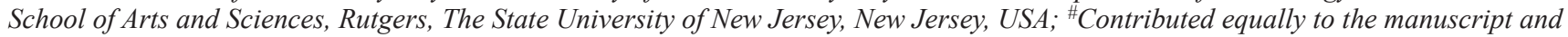
should be listed as shared first author.
\end{abstract}

\begin{abstract}
Curcumin is a polyphenol present in turmeric and is credited with anti-inflammatory, antioxidant, and chemoprotective properties. Questions remain surrounding curcumin's bioavailability and the mechanism by which it may exert neuroprotective effects. Following PRISMA 2009 guidelines, a systematic review was conducted to identify randomized, placebo-controlled trials investigating the effects of curcumin supplementation on cognitive function in older adults (>50 years). Five databases were searched (CINAHL, Cochrane Library, PubMed, SCOPUS, Web of Science) with five studies identified, each using different forms of curcumin and validated cognitive screening measures, meeting inclusion criteria. Curcumin doses ranged from between 90 and 4,000 mg/day, with significant improvements found in three of the five studies. Firstly, the most recent study found improvements with $90 \mathrm{mg}$ of curcumin twice daily in tests of selective reminding $(p=0.002)$, visual memory $(p=0.01)$, and attention $(p<$ 0.0001 ) over 18 months in non-demented individuals. The second study found improvement in Montreal Cognitive Assessment tool with $1,500 \mathrm{mg} /$ day curcumin over 52 weeks $(p=0.02)$. Another study found improvement in serial three subtraction task responses after 4 weeks compared with the placebo group $(p=0.044)$. Of the adverse events reported $(n=58)$, gastrointestinal symptoms were most common $(n=34)$. Before curcumin can be recommended to treat or reduce rates of cognitive decline, well-designed trials with standardization in dose, method of assessing cognition, and duration, are required to determine the most bioavailable form of curcumin with minimal adverse effects.
\end{abstract}

Keywords: Curcumin; Cognition; Dementia; Alzheimer's disease; Dietary supplements; Nutraceuticals.

Abbreviations: A $\beta$, amyloid-beta; AD, Alzheimer's disease; FDDNP-PET, 2-(1-(6[(2-[F-18]fluoroethyl)(methyl)amino]-2-naphthylethylidene)malononitrile positron emission tomography; MCI, mild cognitive impairment; MMSE, Mini-Mental State Examination; MoCA, Montreal Cognitive Assessment; RCT, randomized controlled trial.

Received: December 18, 2018; Revised: March 05, 2019; Accepted: March 06, 2019 *Correspondence to: Nenad Naumovski, Collaborative Research in Bioactives and Biomarkers (CRIBB) Group, P.O. Box 5018, University of Canberra, Bruce, ACT, 2617, Australia. Tel: +61 2 62068719; Fax: +61 2 62015999; E-mail: nenad.naumovski@canberra.edu.au

How to cite this article: Seddon N, D'Cunha NM, Mellor DD, McKune AJ, Georgousopoulou EN, Panagiotakos DB, Kellett J, Naumovski N. Effects of Curcumin on Cognitive Function-A Systematic Review of Randomized Controlled Trials Exploratory Research and Hypothesis in Medicine 2019;4(1):1-11. doi: 10.14218/ ERHM.2018.00024.
Introduction

The rapid global growth in our ageing population has resulted in an increase in neurodegenerative conditions, such as dementia, which results in cognitive decline, loss of functional capacity and reduced quality of life. ${ }^{1}$ However, subtle cognitive changes associated with ageing are common even in the absence of dementia or mild cognitive impairment (MCI). ${ }^{2}$ The maintenance of cognitive function is vital for healthy ageing and daily functioning. Cognitive functions including memory, attention, processing speed, visuospatial perception, and problem-solving decline with ageing and are impacted by both non-modifiable and modifiable risk factors. ${ }^{3,4}$ Nonmodifiable risk factors include age, sex, and genetics, influencing the likelihood of impaired cognitive function, while modifiable risk factors include lifestyle, education, and disease management, 
such as diabetes mellitus and hypertension. ${ }^{2-4}$ There is increasing recognition that addressing modifiable risk and lifestyle factors can preserve cognitive function later into life, potentially delaying the onset of dementia.

While the prevalence of dementia, particularly its most common form of Alzheimer's disease (AD), may be stabilizing in some countries, cases of younger-onset AD are increasing, and the global rate of people living with dementia has more than doubled from 1990 to $2016 .^{5,6}$ Declining cognitive function, memory and behavior are indicative of progressive $\mathrm{AD}$, and at a physiological level, $\mathrm{AD}$ is characterized by the formation of amyloid plaques and neurofibrillary tangles in brain tissue, which contribute to neurodegeneration by blocking cell-signaling activity. ${ }^{7,8}$ The amyloidbeta (A) protein is the main component of the plaques, which are formed in response to cellular degeneration. ${ }^{9}$ Hyperphosphorylated tau proteins are the main component of neurofibrillary tangles, and increased tau synthesis is similarly initiated by cellular degeneration. ${ }^{9}$ The neurodegeneration and presence of abnormal physiological structures are intermediary stimulants for neuroinflammation..$^{10}$ The inhibition of and tau formation, and their subsequent disestablishment of plaques and neurofibrillary tangles is a current target for pharmacological interventions, which to date have proven to be mostly unsuccessful. ${ }^{11}$

Established treatments for AD include cholinesterase inhibitors, such as galantamine, rivastigmine and donepezil, which are widely prescribed to treat symptoms of cognitive decline. However, difficulty in achieving a correct dosage, the timing of intervention, and potential adverse events limit the overall effectiveness of such treatments. ${ }^{12}$ Therefore, non-pharmacological treatments, such as dietary supplements and nutraceuticals, have come into focus for the potential treatment of neurodegenerative conditions. ${ }^{13}$ In particular, plant polyphenols are increasingly being investigated for their potential in the prevention or treatment neurodegenerative conditions, due to their pleotropic activities in the human body. ${ }^{14}$

Polyphenolic compounds, such as curcumin, are being acknowledged for their associations with various beneficial health effects. In particular, the consumption of foods containing polyphenol-rich turmeric (Curcuma longa), such as curry, is associated with reduced incidence of cognitive decline. In 2006, a cross-sectional study of older people found that participants who 'occasionally' and 'often or very often' consumed curry had higher Mini-Mental State Examination (MMSE) scores compared to those who 'never or rarely' consumed curry. ${ }^{15}$ Turmeric supplementation has been associated with alleviation of incontinence, improved recognition of family, and reductions in agitation, anxiety and irritability in people with dementia. ${ }^{16}$ Together, these findings have led to a greater examination of the primary bioactives present in turmeric.

Curcumin is the primary polyphenolic compound of turmeric, a bright yellow-colored spice widely used as a flavor and coloring agent in gastronomy. ${ }^{8,17}$ The term 'curcuminoid' is used to describe the functional group of biochemical agents found in turmeric, of which curcumin is the most abundant, while demethoxycurcumin, bisdemethoxycurcumin and cyclic curcumin forms are less common occurring curcuminoids of turmeric. ${ }^{17}$ Today, curcumin is recognized for its potential to contribute to improved outcomes in the treatment of metabolic syndrome, ${ }^{18}$ diabetes mellitus, ${ }^{19}$ pain, ${ }^{20}$ arthritis, ${ }^{21}$ and symptoms of depression and anxiety. ${ }^{22}$

The health benefits of curcumin are reported to be due to its anti-inflammatory, ${ }^{23-25}$ antioxidant and chemoprotective properties, ${ }^{25-27}$ suggesting that curcumin may also have a role in the prevention of $\mathrm{AD}$. Mechanistically, curcumin has been shown to inhibit the formation of amyloid-beta $(\mathrm{A} \beta)$ fibrils, de-establish preformed fibrils and inhibit tau phosphorylation in in vivo and in vitro animal models. ${ }^{28-31}$ Moreover, transgenic mouse models of $\mathrm{AD}$ have indicated that supplementation with curcumin lowers oxidized proteins and inflammatory cytokines in the brain, ${ }^{32}$ prevents memory deficits, ${ }^{33}$ cognitive decline and suppresses behavioral deficits. ${ }^{34,35}$ However, the evidence supporting curcumin supplementation in humans to prevent or delay cognitive decline remains unclear.

Numerous curcumin formulations aimed to enhance its bioactivity and absorbability have been developed, which may enhance neuroprotective effects due to the ability to cross the blood-brain barrier. However, the debate remains as to whether curcumin promotes brain health and cognitive function through its activity in the brain, or systemically through its effects on gastrointestinal health possibly via interactions with the microbiome. ${ }^{36,37}$ This systematic review aims to assess the current evidence surrounding curcumin from human randomized controlled trials (RCTs) and its effects on cognitive function.

\section{Methods}

The review was preregistered with PROSPERO (Registration: CRD42017084518; https://www.crd.york.ac.uk/prospero/display_record.php?RecordID=84518) in accordance with the Preferred Reporting Items for Systematic Reviews and Meta-analyses (PRISMA) statement for systematic reviews. ${ }^{38}$ The Population, Intervention, Comparator, Outcomes and Setting (PICOS) systematic review approach was used to guide the systematic review. The criteria within each of these categories were as follows:

- Population: Human populations aged 50 years or over that were either healthy older people, people with subjective memory complaints, MCI or a diagnosed form of dementia;

- Intervention: RCTs investigating the effects of curcumin supplementation for 4 weeks or longer;

- Comparator: Human comparators were required, using a placebo or control intervention;

- Outcomes: Human data were analyzed according to validated cognitive measures, biochemical measures, and adverse effects reported; and

- Setting: Any.

\section{Literature search}

Literature searches were conducted across five electronic databases (CINAHL, Cochrane Library, PubMed, SCOPUS and Web of Science) for studies published from January 2000 to November 2017. Searches were re-run again in August 2018, with two additional articles undergoing full-text review, and only one article meeting the inclusion criteria. ${ }^{39}$ The literature search intended to identify the highest quality evidence from double-blind, placebocontrolled, human clinical trials investigating the effect of curcumin supplementation on cognitive function in older adults. Searches were performed using the following search terms "curcumin*", "curcuma", "Curcumin longa", "turmeric", "bisdemethoxycurcumin", "desmethoxycurcumin", "cyclic curcumin" and "cognit*" as well as "elder*", "geriatric", "older", "aged" in conjunction with "Alzheimer's disease", "Alzheimer*", "dementia", "neurocognitive impairment", "mild cognitive impairment", "memory loss", using Boolean operators AND and OR; the (*) indicates the use of wildcard operators. Further studies of interest were identified through examination of the reference lists of relevant studies and previously published review articles. A summary of the search 


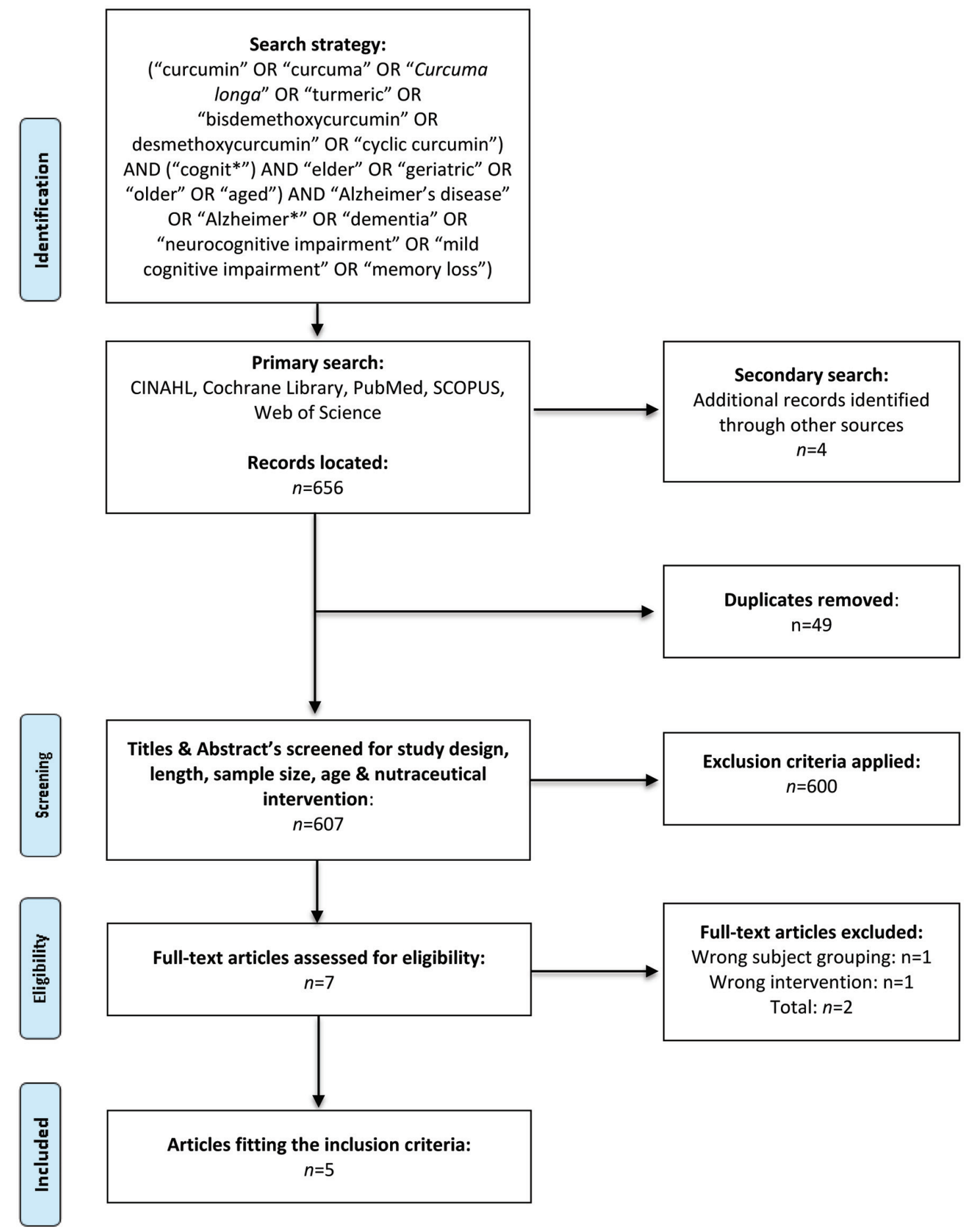

Fig. 1. Search strategy and journal selection process flowchart.

strategy can be found in Figure 1.

\section{Selection criteria}

Peer-reviewed journal articles of studies conducted in humans and published in the English language after January 2000 were eligible for inclusion. Studies investigating participants with a mean age over 50 years were of interest to this review due to the increased likelihood of impaired cognitive function. Studies were included if curcumin supplementation was completed for a minimum duration of 4 weeks to assess effects on symptoms of cognitive decline. 
Table 1. Assessment of bias risk in reviewed studies

\begin{tabular}{|c|c|c|c|c|c|}
\hline Bias classification & Baum et al. ${ }^{44}$ & Ringman et al. ${ }^{42}$ & Cox et al..$^{43}$ & Rainey-Smith et al. ${ }^{41}$ & Small et al. ${ }^{39}$ \\
\hline $\begin{array}{l}\text { Random sequence } \\
\text { generation }\end{array}$ & Low & Low & Low & Low & Low \\
\hline $\begin{array}{l}\text { Allocation } \\
\text { concealment }\end{array}$ & Low & Low & Low & Low & Low \\
\hline $\begin{array}{l}\text { Blinding of } \\
\text { participants and } \\
\text { personnel }\end{array}$ & Unclear & Low & Unclear & Low & Low \\
\hline $\begin{array}{l}\text { Blinding of outcome } \\
\text { assessment }\end{array}$ & Unclear & Low & Unclear & Low & Low \\
\hline $\begin{array}{l}\text { Incomplete } \\
\text { outcome data }\end{array}$ & Low & Low & Low & Low & Low \\
\hline Selective reporting & Low & Low & Low & Low & Low \\
\hline Other bias & High & Low & Low & Low & Low \\
\hline Country & Hong Kong & USA & Australia & Australia & USA \\
\hline Funding source & $\begin{array}{l}\text { University research } \\
\text { grant/Private grant }\end{array}$ & $\begin{array}{l}\text { Research } \\
\text { foundation/Research } \\
\text { grant/government/ } \\
\text { Private institution }\end{array}$ & $\begin{array}{l}\text { Private } \\
\text { institution }\end{array}$ & $\begin{array}{l}\text { Research foundation/ } \\
\text { Research grant }\end{array}$ & $\begin{array}{l}\text { Research foundation/ } \\
\text { Research grant/ } \\
\text { Private institution/ } \\
\text { Private company }\end{array}$ \\
\hline
\end{tabular}

The primary outcomes of interest were studies reporting cognitive function outcomes via validated cognitive screening methods (e.g., MMSE, Montreal Cognitive Assessment (MoCA), Alzheimer's Disease Assessment Scale-cognitive subscale (ADAS-cog)) or diagnosis of a form of dementia. The secondary outcomes were biochemical markers, including A plasma levels, t-tau, p-tau, and lipids. Biomarkers were used to evaluate the association between curcumin supplementation and changes in cognitive function. Adverse effects were also of interest to examine the safety of the curcumin supplements used.

Two researchers (N.S. and N.M.D.) independently conducted the searches. Using the eligibility criteria, both authors first screened based on titles and abstracts. For any articles where this was unclear, the article was included in the full-text review. The full text of the remaining papers was independently reviewed by both authors, and eligible studies were included in the review. Disagreements were discussed to reach consensus with mediation by a third researcher (N.N.), when appropriate.

\section{Data extraction and risk of bias assessment}

The included articles were extracted (N.S.) and checked for consistency (N.M.D.). Data extraction was completed for study design, descriptive statistics, type, dose, and timing of the intervention, and results of the primary and secondary outcomes. The Cochrane Risk of Bias Tool was used to assess the potential for bias by two researchers (N.S. and N.M.D.), ${ }^{40}$ as it is established as the most appropriate assessment tool due to its specificity for bias in RCTs (Table 139,41-43). Disagreements were discussed with a third researcher (E.N.G). Country of study and funding source were also deemed suitable additions to assess the risk of bias.

\section{Results}

A total of 652 studies were identified during the initial elec- tronic database search. Four additional articles were located by manual searching $(n=4)$. After the removal of articles due to duplicates $(n=49)$, inappropriate population $(n=205)$, incorrect study design $(n=333)$ and insufficient intervention $(n=$ 62 ), seven studies remained for full-text assessment. Five trials met the inclusion criteria with the remaining two excluded due to investigating turmeric and curry consumption. ${ }^{22,39,41-45}$ All included studies were randomized, placebo-controlled, doubleblind, clinical trials.

\section{Study characteristics}

The total number of participants enrolled in the included studies was 292, with sample sizes ranging from 34 to 96 participants (Table 2). The primary outcome for all five studies was validated screening measures of cognitive function, while all also measured biochemical outcomes, ${ }^{39,41-44}$ and four reported adverse events (Table 3). ${ }^{39,41,42,44}$ The mean age range of participants was 53.5 to 85.5 years of age. The combined average age of participants was 69.9 years, with three studies assessing Caucasian individuals, ${ }^{41-43}$ one study assessing ethnic Chinese individuals and one study that did not define racial demographics. ${ }^{39,44}$ Three of the studies included participants experiencing cognitive decline. Two studies included healthy participants, ${ }^{41,43}$ two recruited participants with mild to moderate $\mathrm{AD},{ }^{42,44}$ and one included individuals with MCI. ${ }^{39}$ Duration of curcumin supplementation ranged from 4 weeks to 78 weeks.

The included studies used a range of cognitive screening measures. Two studies used the MoCA to evaluate global cognition, ${ }^{39,41}$ two used the MMSE, ${ }^{42,44}$ and one used the ADAS-cog. ${ }^{42}$ Additional measures for executive functioning were used in three studies, ${ }^{39,41,43}$ visual processing in three studies, ${ }^{39,41,43}$ verbal memory in two studies, ${ }^{39,41}$ and delayed recall in two studies. ${ }^{39,43}$ Due to the small number of articles identified, and considerable heterogeneity across studies, we did not deem the inclusion of a meta-analysis to be appropriate at this time. 


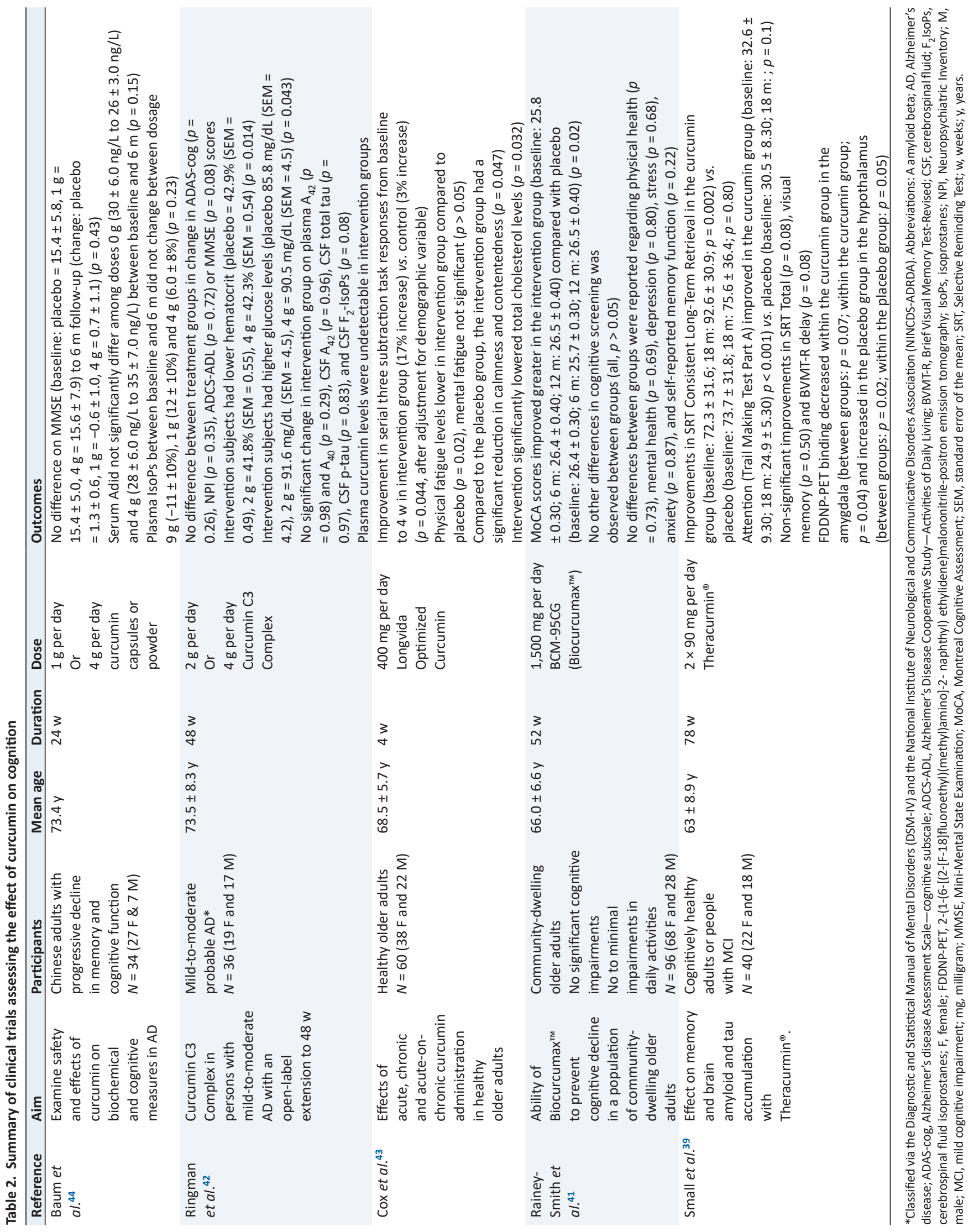


Table 3. Adverse events reported

\begin{tabular}{llll}
\hline Adverse Event & Reference & Curcumin, $\boldsymbol{n}$ (\%) & Placebo, $\boldsymbol{n}$ (\%) \\
\hline Gastrointestinal symptoms & Baum et al. ${ }^{44}$ & $4(11.8)$ & 0 \\
& Rainey-Smith et al. ${ }^{41}$ & $23(0)^{*}$ & 0 \\
& Ringman et al. ${ }^{42}$ & $3(8.3)$ & 0 \\
Edema & Small et al. ${ }^{39}$ & $4(19)$ & $2(10.5)$ \\
Fall or dizziness & Baum et al. ${ }^{44}$ & $1(2.9)$ & $1(2.9)$ \\
Hearing impairment & Baum et al. ${ }^{44}$ & $2(5.9)$ & $1(2.9)$ \\
Respiratory tract infection & Baum et al. ${ }^{44}$ & 0 & $1(2.9)$ \\
Dysphagia like symptoms & Baum et al. ${ }^{44}$ & $1(2.9)$ & $2(5.9)$ \\
\hline
\end{tabular}

*Participants' results excluded from the final statistical analysis.

\section{Dosage and type of curcumin supplementation}

The dosage of supplemental curcumin ranged from $0.09 \mathrm{~g}$ to $4 \mathrm{~g}$ per day, and four studies used a bioenhanced curcumin supplement while the study by Baum et al. used pure curcumin at two different dosages ( $1 \mathrm{~g} /$ day or $4 \mathrm{~g} /$ day) (Table 2$).{ }^{39,41-44}$ One study used Curcumin C3 Complex ${ }^{\circledR}(2$ or $4 \mathrm{~g} /$ day), a combination of curcumin, bisdemethoxycurcumin and demethoxycurcumin standardized for minimum $95 \%$ curcuminoids. ${ }^{42}$ The study by Cox et al..$^{43}$ used Longvida $^{\circledR}$ curcumin $(400 \mathrm{mg} /$ day), an encapsulated lipophilic formulation with $80 \mathrm{mg}$ of curcumin. Rainey-Smith et al..$^{41}$ used BCM- $955^{\circledR} \mathrm{CG}$ (Biocurcumax $\left.{ }^{\mathrm{TM}}\right)(1.5 \mathrm{~g} /$ day) containing $88 \%$ total curcuminoids (curcumin, bisdemethoxycurcumin, demethoxycurcumin and $7 \%$ volatile oils from Curcuma longa) and the trial by Small et al. ${ }^{39}$ used Theracurmin ${ }^{\mathrm{TM}}(2 \times 90 \mathrm{mg} /$ day $)$, a nanoencapsulated submicron crystal solid dispersion of curcumin. The trial by Baum et al. ${ }^{44}$ included $120 \mathrm{mg}$ of Ginkgo leaf extract in both the two curcumin and comparator groups as a standard treatment for $\mathrm{AD}$. The study by Ringman et al..$^{42}$ also permitted vitamin $\mathrm{E}$ supplementation up to 2,000 i.u./day and vitamin $\mathrm{C}$ up to $0.5 \mathrm{~g}$ per day. All studies included a placebo group who were treated in an identical manner to the intervention group but were given capsules, ${ }^{39,41-43}$ or packets of powder that contained no active ingredients and were identical in appearance to those provided to the intervention group. ${ }^{44}$

\section{Results of cognitive outcomes}

All five included studies reported on an outcome of cognitive function (Table 2), with varying results. A study by Baum et al. ${ }^{44}(n=$ 34 ) found no difference between the intervention groups and control groups in the MMSE at the 6-month follow up $(p=0.43)$. The trial by Ringman et al. ${ }^{42}(n=36)$ showed no difference between groups in the ADAS-cog $(p=0.26)$, NPI $(p=0.35)$ or ADCS-ADL $(p=0.72)$. However, the intervention groups performed worse than the placebo group on the MMSE (placebo $=23.2$ vs. $2 \mathrm{~g}=21.4$ and $4 \mathrm{~g}=22.8 ; p=0.08$ ). The three more recent trials used bioenhanced versions of curcumin.

In 2015, Cox et al. ${ }^{43}(n=60)$ used a liposomal encapsulated form of curcumin (Longvida ${ }^{\circledR}$ ) and reported improvements on measures of sustained attention and working memory compared with placebo over a 4-week duration. The curcumin group had a $17 \%$ nonsignificant increase in the number of correct responses in the serial three subtraction task compared to baseline, whereas the placebo group only improved by $3 \%$. However, when the results were adjusted for demographic variables, the effect was significant $(p=0.044)$, suggesting the intervention improved working memory, psychomotor speed and attention when controlling for education and work status.

In 2016, Rainey-Smith et al. ${ }^{41}(n=96)$ reported a time-bytreatment group interaction in the MoCA with Biocurcumax ${ }^{\mathrm{TM}}$ supplementation over 52 weeks compared to the placebo group $(p=0.02)$. However, no other differences between groups were observed in cognition according to the Rey Auditory Verbal Learning Test (all, $p>0.05)$, Wechsler Digit Symbol Scale $(p=0.91)$, or Controlled Oral Word Association Task, for both a noncomputerized $(p=0.09)$ and computerized composite score $(p=0.46)$.

The most recent study by Small et al. ${ }^{39}$ showed improvement from baseline to 18 months in the Buschke-Fuld Selective Reminding Test of verbal memory for the group by time interaction and the total score (both, $p=0.002$ ), with no change in the control group following 18 months of Theracurmin supplementation. Only the intervention group displayed improvements in visual memory (Brief Visuospatial Memory Test-Revised) from baseline to 18 months $(p=0.01)$. The Theracurmin group also improved in attention (Trail Making Test A) after 18 months compared with placebo $(p<0.0001)$. However, it is important to note that both groups exhibited improvements in their verbal memory score at 6 months, representing a potential learning effect. With the Theracumin group performing better at 18 months, the results suggest that curcumin supplementation prevented a decline in cognition compared with the control group. Overall, these results suggest greater improvements in cognitive function with more recent trials using bioenhanced versions of curcumin.

\section{Biochemical outcomes}

Four of the five studies provided results on the outcomes of different biochemical markers, with varying results. ${ }^{39,42-44}$ Baum et $a l .{ }^{44}$ reported that serum A did not differ by dosage of curcumin, and plasma isoprostanes did not change by dosage from baseline to 6 months $(p=0.23)$. Ringman et al. ${ }^{42}$ reported that the intervention groups had lower hematocrit than the control group (control $=42 \%, 2 \mathrm{~g}=41.8 \%, 4 \mathrm{~g}=42.3 \% ; p=0.014)$ and the intervention groups also had higher blood glucose levels (control $=85.9 \mathrm{mg}$ / $\mathrm{dL}, 2 \mathrm{~g}=91.6 \mathrm{mg} / \mathrm{dL}, 4 \mathrm{~g}=90.5 \mathrm{mg} / \mathrm{dL} ; p=0.043$ ) than the control group. There were no significant changes for the intervention groups for $\mathrm{A}_{42}(p=0.98)$ and $\mathrm{A}_{40}(p=0.29), \operatorname{CSF} \mathrm{A}_{42}(p=0.96)$, 
CSF total tau $(p=0.97)$, CSF $p$-tau $(p=0.83)$, and CSF $\mathrm{F}_{2}$ - isoprostanes $(p=0.08)$. The study by Cox et al.$^{43}$ showed significantly lower total cholesterol levels in the intervention group compared to the control group $(p=0.032)$. No other significant differences on blood biomarkers were noted regarding IL-1, IL- 6 and tumor necrosis factor $\alpha(\mathrm{TNF} \alpha)$, as participants had serum levels below measurable evaluation. The study by Small et al. ${ }^{39}$ performed 2-(1(6-[(2-[F-18]fluoroethyl)(methyl)amino]-2-naphthylethylidene) malononitrile positron emission tomography (FDDNP-PET) scans on participants at baseline and 18 months. Compared with controls, accumulation of $\mathrm{A} \beta$ and tau was lower in the Theracumin group $(p=0.04)$. FDDNP-PET binding decreased within the curcumin group in the amygdala (between groups: $p=0.07$; within curcumin group; $p=0.04$ ) and increased in the placebo group in the hypothalamus (between groups: $p=0.02$; within placebo group: $p=0.05$ ). They also measured levels of free curcumin, which increased in the intervention group, confirming compliance with supplementation.

\section{Adverse events}

Adverse events were reported in four studies, with one study reporting that the curcumin was well tolerated overall. ${ }^{43} \mathrm{~A}$ total of 40 $(13.7 \%)$ adverse events were reported in the intervention groups compared with only $7(2.40 \%)$ in the control groups. The most common adverse event was gastrointestinal symptoms, reported in four studies, ${ }^{39,41,42,44}$ with a total of 34 individuals given the curcumin intervention reporting experiencing these symptoms. Two studies showed specific symptoms, including black stools, diarrhea, transient abdominal pain, gastritis, and nausea. ${ }^{39,42}$ It is important to note that the 23 intervention participants who reported adverse events in Rainey-Smith et al. ${ }^{41}$ were excluded from the final statistical analysis. Baum et al. ${ }^{44}$ reported additional adverse events in both intervention and placebo groups, including falls or dizziness ( $n=2$ and $n=1$, respectively), edema ( $n=1$ and $n=$ 1 , respectively) and respiratory tract infections ( $n=1$ and $n=2$, respectively). Additionally, one participant in the control group experienced hearing impairment. Two participants in the study by Ringman et al. ${ }^{42}$ experienced difficulty swallowing the capsules. Small et al. ${ }^{39}$ reported six participants with gastrointestinal symptoms (abdominal pain, gastritis and nausea), comprising four from the intervention group and two from the placebo group. Both the studies by Small et al. ${ }^{39}$ and Baum et al. ${ }^{44}$ found adverse events occurring among the control group participants. We can only speculate that these reported events in the control groups were due to the general effects of ageing in populations that may be more susceptible to illness. For the trials of Longvida $\AA$ and Theracurmin ${ }^{\mathrm{TM}}$ that found no or minimal side effects, it is suggested that the issue of gastrointestinal symptoms can be potentially resolved with these forms. ${ }^{39,43}$

\section{Risk of bias assessment}

The reviewed studies indicated low levels of bias among the criteria published in the Cochrane tool ${ }^{40}$ No articles presented with a high, overall risk of overall bias. However, several criteria were deemed as "unclear" for the trials by Baum et al. ${ }^{44}$ and Cox et $a l .{ }^{43}$ As the study by Baum et al. ${ }^{44}$ was published as a letter to the editor, this may explain why sufficient detail was not presented to assess the risk of bias clearly. There were apparent commercial links between four of the five studies. Small et al. ${ }^{39}$, Ringman et $a l .{ }^{42}$, Rainey-Smith et $a l^{41}$ and Cox et al. ${ }^{43}$ all obtained their in- tervention products from private companies and these had been provided free of charge. The trials by Ringman et al. ${ }^{42}$ and Cox et $a l .{ }^{43}$ received direct funding from these organizations, which were acknowledged in the cited papers.

\section{Discussion}

This is the first systematic review of curcumin supplementation and its association with cognitive function. Together, the results suggest that various formulations of curcumin supplements may improve performance on some cognitive domains in older people and that supplementation may improve biochemical markers associated with AD. Three of the five studies found improvement in cognitive function when supplementing with curcumin. Two studies found improvements in MoCA scores and serial three subtraction task response, respectively. ${ }^{41,43}$ However, in the most recent article included in this systematic review, improvements were observed using a new nanoparticle formulation of curcumin in measures of memory and attention, and through less accumulation of $\mathrm{A} \beta$ and tau proteins in the hypothalamus and amygdala. ${ }^{39}$ This study by Small et al. ${ }^{39}$ was also the longest trial to date, with a total time of 18 months. However, when assessing the studies together, it is difficult to generalize the results due to considerable heterogeneity across trials and the low number of participants overall. The major limitation of this systematic review, and curcumin research investigating its effects on cognitive function in general, is the small number of clinical trials conducted to date.

Supplementation dosage and duration of intervention greatly differed amongst the included studies. The earliest studies, those by Baum et al. ${ }^{44}$ and Ringman et al.., ${ }^{42}$ used between 1-4 g/day curcumin, and the study by Rainey-Smith et al.,${ }^{41}$ which resulted in considerable adverse gastrointestinal symptoms, used $1.5 \mathrm{~g} /$ day. However, the two studies using bioenhanced versions used much smaller doses. ${ }^{39,43}$ Cox et al ${ }^{43}$ used 0.4 g/day of Longvida ${ }^{\circledR}$, which contains only $0.08 \mathrm{~g}$ of actual curcumin. Small et al ${ }^{39}$ provided $0.09 \mathrm{~g}$ of Theracumin twice per day. The development of bioenhanced versions of curcumin resulted in lower dosages in the studies by Cox et al. ${ }^{43}$ and Small et al., ${ }^{39}$ which appear to reduce the likelihood of gastrointestinal distress due to their increased absorption. ${ }^{25}$ The high dosages of low-bioavailable curcumin helps to explain the gastrointestinal effects observed by Baum et al. ${ }^{44}$ and perhaps, the null results reported. Moreover, this study and the study by Ringman et al $^{42}$ were the only studies to assess cognitive function in individuals with an established progressive decline in cognitive function. Plausibly, their gastrointestinal function may have been disrupted already as is often observed in people living with dementia. ${ }^{46}$ The findings in animal models have suggested that there is gut-brain modulation of cognitive function and this represents a novel and under-researched area in humans. ${ }^{25}$ In these two studies, the timing of curcumin supplementation in relation to the disease course may have affected the findings, as progressive cognitive decline was already underway. Nevertheless, gastrointestinal symptoms observed in people living with dementia, consuming newer formulations of curcumin, are yet to be examined.

Curcumin, in its pure form, appears to be poorly tolerated in large dosages ( $>1 \mathrm{~g}$ per day). Participants who consumed more than $1 \mathrm{~g}$ per day reported some adverse events manifested as gastrointestinal symptoms. Similar adverse events have been reported in other clinical trials of curcumin. In a systematic review of RCTs of treatment of joint arthritis, Daily et al. ${ }^{21}$ reported six studies that found curcumin leading to gastrointestinal complaints. Interestingly, joint pain occurrence had a $2.5 \%$ decrease per gram of curcum- 
in, yet it was accepted that curcumin doses were considered safe if they did not exceed $1.2 \mathrm{~g}$ per day for a period of 4 months. ${ }^{21}$ As such, the dosages prescribed in three of the five studies included in this review would not be deemed safe. Based on current recommendations, only the Longvida ${ }^{\circledR}$ and Theracumin studies provided safe dosage, ${ }^{39,43}$ from among the five included trials. Nevertheless, Cheng et al. ${ }^{47}$ examined the chemoprotective properties of curcumin on high risk or premalignant lesions in patients with a bioenhanced curcumin with dosages as high as $12 \mathrm{~g}$ per day and reported no toxicity in dosages up to $8 \mathrm{~g}$ per day. Therefore, the tolerability of specific doses of the different formulations of curcumin needs to be assessed.

It is well established that curcumin has relatively low bioavailability, low intestinal absorption, and rapid liver metabolism, with the majority being excreted through the faces in 3-6 h. ${ }^{48}$ This has led to the development of bioenhanced formulations used in recent trials with Longvida ${ }^{\circledR}$ that found as much as 100 times more bioavailability than naturally occurring curcumin. ${ }^{49}$ Rainey-Smith et al. ${ }^{41}$ supplemented with BCM-95 CG (Biocurcumax), which is reported to be 6.93 times more bioavailable than pure curcumin purportedly due to the noncurcuminoid compounds. ${ }^{50}$ Ringman et $a l .{ }^{42}$ and Cox et $a l .{ }^{43}$ also utilized manufactured curcumin supplements Curcumin $\mathrm{C} 3$ Complex, and Longvida ${ }^{\circledR}$ Optimized Curcumin, respectively. The study by Small et al. ${ }^{39}$ measured the kinetics of curcumin absorption over $6 \mathrm{~h}$ and found Theracurmin to lead to higher peak concentrations compared with Meriva curcumin and a standardized turmeric extract.

Considerable heterogeneity was seen across trials included in this systematic review, with different curcumin formulations, dosages and cognitive outcomes used. For these reasons, a meta-analysis is not currently considered suitable for this data. Improved standardization of study measures in trials may render this feasible in the future. The length of studies also varied with the study by Cox et $a l .{ }^{43}$ running for 4 weeks, making it difficult to extrapolate the results to the same extent as the longer trials. However, this group is currently testing Longvida ${ }^{\circledR}$ in a 12 week trial with improvements in working memory reported recently in an RCT of older Australians (unpublished results). ${ }^{51}$ While four studies measured biochemical outcomes, there was no consistency in the markers of interest that were measured. Two studies conducted APOE genotyping. ${ }^{39,41}$ The APOE gene has been identified as the most recognized genetic risk for $\mathrm{AD}$ and could impact the results through unknown mechanisms. ${ }^{52}$ Also, none of the three most recent trials investigated older people that had existing cognitive impairments. This review evaluated both healthy individuals and those with cognitive impairment. Future studies investigating curcumin in both populations of older people are needed to assess curcumin from both a preventative and treatment perspective.

There has been a substantial increase in the number of clinical trials investigating curcumin to treat or prevent a range of conditions in recent years. The studies included in the present systematic review were conducted within the last 10 years, indicative of the modern ideology of natural medicine and a response to the lack of new pharmacological treatments for cognitive decline and dementia prevention. This contemporary trend in natural medicine will no doubt result in further human clinical trials, potentially driven by commercial interest and industry funding. Furthermore, curcumin interventions may be more efficacious if the supplementation is given earlier in life as a preventative measure, rather than a curative (or palliative) measure. In 2018, results from RCTs of curcumin improved symptoms associated with rheumatoid arthritis and liver dysfunction. ${ }^{53,54}$ Curcumin has also shown promise in improving the metabolic profile of diabetes mellitus, a risk factor for the development of AD. ${ }^{19}$ In recent clinical trials of people with diabetes mellitus, curcumin has led to improvements in the lipid profile, fasting blood glucose, antioxidant status, and inflammatory markers, suggesting that curcumin may be useful as a strategy to reduce the risk of AD related risk factors earlier in life. ${ }^{19}$

Considerable attention has been given to curcumin's bioavailability and testing of new formulations, including several commercially available, proprietary formulations not used in the reviewed studies, such as Meriva ${ }^{\circledR}$ (a phytosomal formulation with curcuminoids and lecithin) and CW8 (y-cyclodextrin). ${ }^{49}$ A recent review of curcumin formulation bioavailability compared 11 different formulations, identified as NovaSol ${ }^{\circledR}$ (Micellized with Tween-80), CurcuWin $^{\circledR}$ (Water-soluble formulation) and Longvida ${ }^{\circledR}$ possessing the highest bioavailability relative to pure curcumin. However, while the cell signaling mechanisms have been extensively investigated, dietary polyphenols are starting to come into focus for their benefits, irrespective of low bioavailability. Findings from animal studies have shown that curcumin may impact gut health through the microbiome by the promotion of bacterial diversity, reduced gut permeability and improved tight junction function; all of these contribute to a reduction in inflammation..$^{36}$ More research is needed to understand how curcumin exerts protection through modulation of the gut-brain axis.

Curcumin is only one of four curcuminoids present in turmeric, being the most abundant and most well studied. The potential capacity of curcumin as an antioxidant, anti-inflammatory and health promotive agent are far greater than its less known curcuminoids. ${ }^{55-57}$ However, lesser-known curcuminoids, such as bisdemethoxycurcumin and desmethoxycurcumin, may also provide health benefits. Furthermore, research will assess the potential for benefits to humans of specific curcumin analogues, such as J147 (which has been shown to reverse cognitive impairment in mice by targeting mitochondrial ATP synthase) and EF24 (which is being explored as a novel senolytic agent)..$^{58-60}$

The effects of curcumin on improving cognitive function in combination with other nutraceuticals also represent an important future area for investigation. ${ }^{25}$ Recently, curcumin was tested in combination with Huperzine A in an open-label study of individuals with dementia and MCI. ${ }^{61}$ A small study $(n=15)$ found improvements in the ADAS-cog (Japanese version) after 12 and 28 weeks with only $60 \mathrm{mg}$ curcumin with $50 \mu \mathrm{g}$ active Huperzine A, $3 \mathrm{mg}$ piperine and other potentially active ingredients. Trials are ongoing, investigating the impact of curcumin in combination with omega- 3 fatty acids to promote cerebral endothelial vasodilator function synergistically. ${ }^{62}$ Curcumin is also being tested in conjunction with a lifestyle intervention involving yoga. ${ }^{63}$ Combining curcumin with compounds that cross the blood-brain barrier, such as beta-hydroxybutyrate, may enhance its effects centrally. ${ }^{64}$ Finally, the design of food products or delivery systems that are targeted to specific sites of the small intestine (upper portion of the jejunum) may improve the efficacy of curcumin interventions. Similar to other polyphenolic compounds, absorption rates may differ depending on the method of oral delivery. ${ }^{65}$

\section{Future research prospect}

Curcumin's pleiotropic effects are becoming more widely understood; however, the mechanisms by which it exerts its benefits to cognitive function remain unclear. Curcumin and other polyphenols have low bioavailability but are known to be recycled and reabsorbed, delivering improvements in gut health, and a reduction in systemic inflammation with chronic consumption. To properly 
evaluate the effects of curcumin on cognitive function, future acute and long-term trials should be conducted assessing the efficacy of different formulations of curcumin in crossover trials, analyzing by APOE genotype, ${ }^{13}$ and controlling for potential gut dysbiosis pre-randomization. A range of other biochemical measures will provide insight into curcumin's benefits, such as microbiome testing, homocysteine, C-reactive protein and other proinflammatory cytokines, and markers of mitochondrial function.

\section{Conclusions}

In this first systematic review to evaluate the effects of curcumin and cognitive function, the results suggest that novel formulations of curcumin are promising strategies to promote cognitive function. However, despite trials of up to 18 months, there is insufficient evidence to support curcumin supplementation as an effective means of both preventing and treating dementia and symptoms of cognitive decline. The current evidence in humans is promising but generalizability of the results is limited by the small number of trials, relatively small sample sizes, and the considerable variation across studies. Future research may focus on examining the role of bioavailability to further elucidate the mechanisms by which curcumin exerts its pleiotropic effects, through examination of the gut-brain axis and relevant biomarkers.

\section{Acknowledgments}

Nathan M. D'Cunha is supported by a Dementia Australia Research Foundation $\mathrm{PhD}$ scholarship. All other authors have no sources of funding to declare.

\section{Conflict of interest}

The authors have no conflict of interests related to this publication.

\section{Author contributions}

$\mathrm{NN}$ and NMD designed the study and devised the research questions. NMD and NS drafted the manuscript and carried out the literature review and data extraction. NN, NS and NMD were in charge of editing and formatting the final manuscript. NS, NMD, ENG, DP, JK, AJM, DDM and NN contributed to the writing and provided critical feedback. All authors read and contributed to the final manuscript.

\section{References}

[1] Livingston G, Sommerlad A, Orgeta V, Costafreda SG, Huntley J, Ames $D$, et al. Dementia prevention, intervention, and care. Lancet 2017;390(10113):2673-2734. doi:10.1016/S0140-6736(17)31363-6.

[2] Harada CN, Natelson Love MC, Triebel KL. Normal cognitive aging. Clin Geriatr Med 2013;29(4):737-752. doi:10.1016/j.cger.2013.07.002.

[3] Klimova B, Valis M, Kuca K. Cognitive decline in normal aging and its prevention: a review on non-pharmacological lifestyle strategies. Clin Interv Aging 2017;12:903-910. doi:10.2147/CIA.S132963.

[4] D'Cunha NM, McKune AJ, Panagiotakos DB, Georgousopoulou EN, Thomas J, Mellor DD, et al. Evaluation of dietary and lifestyle changes as modifiers of S100beta levels in Alzheimer's disease. Nutr Neurosci
2019;22(1):1-18. doi:10.1080/1028415X.2017.1349032.

[5] Zhu X-C, Tan L, Wang H-F, Jiang T, Cao L, Wang C, et al. Rate of early onset Alzheimer's disease: a systematic review and meta-analysis. Ann Transl Med 2015;3(3):38. doi:10.3978/j.issn.2305-5839.2015.01.19.

[6] Nichols E, Szoeke CEI, Vollset SE, Abbasi N, Abd-Allah F, Abdela J, et al. Global, regional, and national burden of Alzheimer's disease and other dementias, 1990-2016: a systematic analysis for the Global Burden of Disease Study 2016. Lancet Neurol 2019;18(1):88-106. doi:10.1016/S1474-4422(18)30403-4.

[7] Hamaguchi T, Ono K, Yamada M. REVIEW: Curcumin and Alzheimer's disease. CNS Neurosci Ther 2010;16(5):285-297. doi:10.1111/ j.1755-5949.2010.00147.x.

[8] Brondino N, Re S, Boldrini A, Cuccomarino A, Lanati N, Barale F, et al. Curcumin as a therapeutic agent in dementia: a mini systematic review of human studies. ScientificWorldJournal 2014;2014:174282. doi:10.1155/2014/174282.

[9] Armstrong RA. The molecular biology of senile plaques and neurofibrillary tangles in Alzheimer's disease. Folia Neuropathol 2009;47(4):289-299.

[10] Akiyama H, Barger S, Barnum S, Bradt B, Bauer J, Cole GM, et al. Inflammation and Alzheimer's disease. Neurobiol Aging 2000;21(3):383-421. doi:10.1016/S0197-4580(00)00124-X.

[11] Yiannopoulou KG, Papageorgiou SG. Current and future treatments for Alzheimer's disease. Ther Adv Neurol Disord 2013;6(1):19-33. doi:10.1177/1756285612461679.

[12] Gill SS, Anderson GM, Fischer HD, Bell CM, Li P, Normand SL, et al. Syncope and its consequences in patients with dementia receiving cholinesterase inhibitors: a population-based cohort study. Arch Intern Med 2009;169(9):867-873. doi:10.1001/archinternmed.2009.43.

[13] D'Cunha NM, Georgousopoulou EN, Dadigamuwage L, Kellett J, Panagiotakos DB, Thomas J, et al. Effect of long-term nutraceutical and dietary supplement use on cognition in the elderly: a 10year systematic review of randomised controlled trials. $\mathrm{Br} J$ Nutr 2018;119(3):280-298. doi:10.1017/\$0007114517003452.

[14] Vauzour D. Polyphenols and brain health. OCL 2017;24(2):A202. doi:10.1051/ocl/2017008.

[15] Ng TP, Chiam PC, Lee T, Chua HC, Lim L, Kua EH. Curry consumption and cognitive function in the elderly. Am J Epidemiol 2006;164(9):898906. doi:10.1093/aje/kwj267.

[16] Hishikawa N, Takahashi Y, Amakusa Y, Tanno Y, Tuji Y, Niwa H, et al. Effects of turmeric on Alzheimer's disease with behavioral and psychological symptoms of dementia. Ayu 2012;33(4):499-504. doi:10.4103/0974-8520.110524.

[17] Priyadarsini KI. The chemistry of curcumin: from extraction to therapeutic agent. Molecules 2014;19(12):20091-20112. doi:10.3390/ molecules191220091.

[18] Panahi Y, Hosseini MS, Khalili N, Naimi E, Majeed M, Sahebkar A. Antioxidant and anti-inflammatory effects of curcuminoid-piperine combination in subjects with metabolic syndrome: A randomized controlled trial and an updated meta-analysis. Clin Nutr 2015;34(6):1101-1108. doi:10.1016/j.clnu.2014.12.019.

[19] Rivera-Mancía S, Trujillo J, Chaverri JP. Utility of curcumin for the treatment of diabetes mellitus: Evidence from preclinical and clinical studies. J Nutr Intermed Metab 2018;14:29-41. doi:10.1016/j. jnim.2018.05.001.

[20] Sahebkar A, Henrotin Y. Analgesic Efficacy and safety of curcuminoids in clinical practice: a systematic review and meta-analysis of randomized controlled trials. Pain Med 2016;17(6):1192-1202. doi:10.1093/pm/pnv024.

[21] Daily JW, Yang M, Park S. Efficacy of turmeric extracts and curcumin for alleviating the symptoms of joint arthritis: a systematic review and meta-analysis of randomized clinical trials. J Med Food 2016;19(8):717-729. doi:10.1089/jmf.2016.3705.

[22] Ng QX, Koh SSH, Chan HW, Ho CYX. Clinical use of curcumin in depression: a meta-analysis. J Am Med Dir Assoc 2017;18(6):503-508. doi:10.1016/j.jamda.2016.12.071.

[23] Sahebkar A, Cicero AFG, Simental-Mendía LE, Aggarwal BB, Gupta SC Curcumin downregulates human tumor necrosis factor- $\alpha$ levels: $A$ systematic review and meta-analysis ofrandomized controlled trials. Pharmacol Res 2016;107:234-242. doi:10.1016/j.phrs.2016.03.026.

[24] Derosa G, Maffioli P, Simental-Mendía LE, Bo S, Sahebkar A. Effect 
of curcumin on circulating interleukin- 6 concentrations: A systematic review and meta-analysis of randomized controlled trials. Pharmacol Res 2016;111:394-404. doi:10.1016/j.phrs.2016.07.004.

[25] D'Cunha NM, Seddon N, Mellor DD, Georgousopoulou EN, McKune AJ, Panagiotakos DB, et al. Curcumin for cognition: is it just hype, based on current data? Adv Nutr 2019;10(1):179-181. doi:10.1093/ advances/nmy066.

[26] Ak T, Gulcin I. Antioxidant and radical scavenging properties of curcumin. Chem Biol Interact 2008;174(1):27-37. doi:10.1016/j. cbi.2008.05.003.

[27] Rahmani AH, Al Zohairy MA, Aly SM, Khan MA. Curcumin: a potential candidate in prevention of cancer via modulation of molecular pathways. Biomed Res Int 2014;2014:761608. doi:10.1155/2014/761608.

[28] Yang F, Lim GP, Begum AN, Ubeda OJ, Simmons MR, Ambegaokar SS, et al. Curcumin inhibits formation of amyloid beta oligomers and fibrils, binds plaques, and reduces amyloid in vivo. J Biol Chem 2005;280(7):5892-5901. doi:10.1074/jbc.M404751200.

[29] Mutsuga M, Chambers JK, Uchida K, Tei M, Makibuchi T, Mizorogi $\mathrm{T}$, et al. Binding of curcumin to senile plaques and cerebral amyloid angiopathy in the aged brain of various animals and to neurofibrillary tangles in Alzheimer's brain. J Vet Med Sci 2012;74(1):51-57. doi:10.1292/jvms.11-0307.

[30] Huang HC, Tang D, Xu K, Jiang ZF. Curcumin attenuates amyloid-betainduced tau hyperphosphorylation in human neuroblastoma $\mathrm{SH}$ SY5Y cells involving PTEN/Akt/GSK-3beta signaling pathway. J Recept Signal Transduct Res 2014;34(1):26-37. doi:10.3109/10799893.2013 .848891.

[31] Miyasaka T, Xie C, Yoshimura S, Shinzaki Y, Yoshina S, Kage-Nakadai $\mathrm{E}$, et al. Curcumin improves tau-induced neuronal dysfunction of nematodes. Neurobiol Aging 2016;39:69-81. doi:10.1016/j.neurobiolaging.2015.11.004.

[32] Lim GP, Chu T, Yang F, Beech W, Frautschy SA, Cole GM. The curry spice curcumin reduces oxidative damage and amyloid pathology in an Alzheimer transgenic mouse. J Neurosci 2001;21(21):8370-8377. doi:10.1523/JNEUROSCI.21-21-08370.2001.

[33] Frautschy SA, Hu W, Kim P, Miller SA, Chu T, Harris-White ME, et al. Phenolic anti-inflammatory antioxidant reversal of Abetainduced cognitive deficits and neuropathology. Neurobiol Aging 2001;22(6):993-1005. doi:10.1016/S0197-4580(01)00300-1.

[34] Ma QL, Yang F, Rosario ER, Ubeda OJ, Beech W, Gant DJ, et al. Betaamyloid oligomers induce phosphorylation of tau and inactivation of insulin receptor substrate via c-Jun $\mathrm{N}$-terminal kinase signaling: suppression by omega-3 fatty acids and curcumin. J Neurosci 2009;29(28):9078-9089. doi:10.1523/JNEUROSCI.1071-09.2009.

[35] Ma QL, Zuo X, Yang F, Ubeda OJ, Gant DJ, Alaverdyan M, et al. Curcumin suppresses soluble tau dimers and corrects molecular chaperone, synaptic, and behavioral deficits in aged human tau transgenic mice. J Biol Chem 2013;288(6):4056-4065. doi:10.1074/jbc. M112.393751.

[36] Lopresti AL. The problem of curcumin and its bioavailability: could its gastrointestinal influence contribute to its overall health-enhancing effects? Adv Nutr 2018;9(1):41-50. doi:10.1093/advances/nmx011.

[37] Carabotti M, Scirocco A, Maselli MA, Severi C. The gut-brain axis: interactions between enteric microbiota, central and enteric nervous systems. Ann gastroenterol 2015;28(2):203-209.

[38] Moher D, Liberati A, Tetzlaff J, Altman DG. Preferred reporting items for systematic reviews and meta-analyses: the PRISMA statement. BMJ 2009;339:b2535. doi:10.1136/bmj.b2535.

[39] Small GW, Siddarth P, Li Z, Miller KJ, Ercoli L, Emerson ND, et al. Memory and brain amyloid and tau effects of a bioavailable form of curcumin in non-demented adults: a double-blind, placebo-controlled 18-month trial. Am J Geriatr Psychiatry 2018;26(3):266-277. doi:10.1016/j.jagp.2017.10.010.

[40] Higgins J, Green S. Cochrane Handbook for Systematic Reviews of Interventions Version 5.1.0 [updated March 2011]. The Cochrane Collaboration; 2011.

[41] Rainey-Smith SR, Brown BM, Sohrabi HR, Shah T, Goozee KG, Gupta VB, et al. Curcumin and cognition: a randomised, placebo-controlled, double-blind study of community-dwelling older adults. $\mathrm{Br} J$ Nutr 2016;115(12):2106-2113. doi:10.1017/S0007114516001203.

[42] Ringman JM, Frautschy SA, Teng E, Begum AN, Bardens J, Beigi M, et al. Oral curcumin for Alzheimer's disease: tolerability and efficacy in a 24-week randomized, double blind, placebo-controlled study. Alzheimers Res Ther 2012;4(5):43. doi:10.1186/alzrt146.

[43] Cox KH, Pipingas A, Scholey AB. Investigation of the effects of solid lipid curcumin on cognition and mood in a healthy older population. J Psychopharmacol 2015;29(5):642-651. doi:10.1177/0269881114552744.

[44] Baum L, Lam CW, Cheung SK, Kwok T, Lui V, Tsoh J, et al. Six-month randomized, placebo-controlled, double-blind, pilot clinical trial of curcumin in patients with Alzheimer disease. J Clin Psychopharmacol 2008;28(1):110-113. doi:10.1097/jcp.0b013e318160862c.

[45] Lee MS, Wahlqvist ML, Chou YC, Fang WH, Lee JT, Kuan JC, et al. Turmeric improves post-prandial working memory in pre-diabetes independent of insulin. Asia Pac J Clin Nutr 2014;23(4):581-591. doi:10.6133/apjen.2014.23.4.24

[46] Ticinesi A, Tana C, Nouvenne A, Prati B, Lauretani F, Meschi T. Gut microbiota, cognitive frailty and dementia in older individuals: a systematic review. Clin Interv Aging 2018;13:1497-1511. doi:10.2147/ CIA.S139163.

[47] Cheng AL, Hsu CH, Lin JK, Hsu MM, Ho YF, Shen TS, et al. Phase I clinical trial of curcumin, a chemopreventive agent, in patients with highrisk or pre-malignant lesions. Anticancer Res 2001;21(4b):28952900.

[48] Kunwar A, Priyadarsini KI. Curcumin and its role in chronic diseases. Adv Exp Med Biol 2016;928:1-25. doi:10.1007/978-3-319-41334-1_1.

[49] Jamwal R. Bioavailable curcumin formulations: A review of pharmacokinetic studies in healthy volunteers. J Integr Med 2018;16(6):367374. doi:10.1016/j.joim.2018.07.001.

[50] Antony B, Merina B, lyer VS, Judy N, Lennertz K, Joyal S. A pilot crossover study to evaluate human oral bioavailability of BCM-95CG (Biocurcumax), a novel bioenhanced preparation of curcumin. Indian J Pharm Sci 2008;70(4):445-449. doi:10.4103/0250-474X.44591.

[51] Scholey AB, Cox KH, Pipingas A, White D, editors. Effects of a highly bioavailable curcumin extract on mood and memory in healthy older adults: A 12 week double-blind, placebo-controlled trial. Nutrition Society of Australia Annual Scientific Meeting; 2018 28th November 2018; Canberra, Australia.

[52] Farrer LA, Cupples LA, Haines JL, Hyman B, Kukull WA, Mayeux $R$, et al. Effects of age, sex, and ethnicity on the association between apolipoprotein $E$ genotype and Alzheimer disease. A meta-analysis. APOE and Alzheimer Disease Meta Analysis Consortium. Jama 1997;278(16):1349-1356. doi:10.1001/ jama.1997.03550160069041.

[53] Amalraj A, Varma K, Jacob J, Divya C, Kunnumakkara AB, Stohs SJ, et al. A novel highly bioavailable curcumin formulation improves symptoms and diagnostic indicators in rheumatoid arthritis patients: a randomized, double-blind, placebo-controlled, two-dose, threearm, and parallel-group study. J Med Food 2017;20(10):1022-1030. doi:10.1089/jmf.2017.3930.

[54] Mohammadi E, Tamaddoni A, Qujeq D, Nasseri E, Zayeri F, Zand H, et al. An investigation of the effects of curcumin on iron overload hepcidin level, and liver function in beta-thalassemia major patients: A double-blind randomized controlled clinical trial. Phytother Res 2018;32(9):1828-1835. doi:10.1002/ptr.6118.

[55] Sandur SK, Pandey MK, Sung B, Ahn KS, Murakami A, Sethi G, et al. Curcumin, demethoxycurcumin, bisdemethoxycurcumin, tetrahydrocurcumin and turmerones differentially regulate anti-inflammatory and anti-proliferative responses through a ROS-independent mechanism. Carcinogenesis 2007;28(8):1765-1773. doi:10.1093/carcin/ bgm123.

[56] Aftab N, Vieira A. Antioxidant activities of curcumin and combinations of this curcuminoid with other phytochemicals. Phytother Res 2010;24(4):500-502. doi:10.1002/ptr.2960.

[57] Somparn P, Phisalaphong C, Nakornchai S, Unchern S, Morales NP. Comparative antioxidant activities of curcumin and its demethoxy and hydrogenated derivatives. Biol Pharm Bull 2007;30(1):74-78. doi:10.1248/bpb.30.74.

[58] Prior M, Dargusch R, Ehren JL, Chiruta C, Schubert D. The neurotrophic compound $\mathrm{J} 147$ reverses cognitive impairment in aged Alzheimer's disease mice. Alzheimers Res Ther 2013;5(3):25. doi:10.1186/ alzrt179.

[59] Goldberg J, Currais A, Prior M, Fischer W, Chiruta C, Ratliff E, et al. 
The mitochondrial ATP synthase is a shared drug target for aging and dementia. Aging Cell 2018;17(2):e12715. doi:10.1111/acel.12715.

[60] Li W, He Y, Zhang R, Zheng G, Zhou D. The curcumin analog EF24 is a novel senolytic agent. Aging (Albany NY) 2019;11(2):771-782. doi:10.18632/aging.101787.

[61] Tabira T, Kawamura N. A study of a supplement containing huperzine a and curcumin in dementia patients and individuals with mild cognitive impairment. J Alzheimers Dis 2018;63(1):75-78. doi:10.3233/ JAD-171154.

[62] Kuszewski JC, Wong RHX, Howe PRC. Can curcumin counteract cognitive decline? Clinical trial evidence and rationale for combining $\omega-3$ fatty acids with curcumin. Adv Nutr 2018;9(2):105-113. doi:10.1093/ advances/nmx013.

[63] ClinicalTrials.gov. Curcumin and yoga therapy for those at risk for Alzheimer's Disease: U.S. National Library of Medicine; 2013 [14th March 2013] Available from: https://clinicaltrials.gov/ct2/show/ NCT01811381.

[64] Achanta LB, Rae CD. $\beta$-Hydroxybutyrate in the brain: one molecule, multiple mechanisms. Neurochem Res 2017;42(1):35-49. doi:10.1007/s11064-016-2099-2.

[65] Naumovski N, Blades LB, Roach DP. Food inhibits the oral bioavailability of the major green tea antioxidant epigallocatechin gallate in humans. Antioxidants (Basel) 20157 4(2):373-393. doi:10.3390/ antiox4020373. 